\title{
Parents' friends and families in neonatal intensive care units: A cross-national qualitative study on staff perceptions and experiences
}

\author{
Renée Flacking PhD, Professor ${ }^{1}$ (1) | Helle Haslund-Thomsen PhD, Associate Professor ${ }^{2}$ | \\ Rakel Jónsdóttir MSc, PhD-candidate ${ }^{3}$ | Sini Poropudas MSc, Home care leader ${ }^{4}$ | \\ Anna Axelin PhD, Associate Professor ${ }^{4}$
}

${ }^{1}$ School of Health and Welfare, Dalarna University, Falun, Sweden

${ }^{2}$ Paediatric Research Unit, Clinical Nursing Research Unit, Aalborg University Hospital, Clinical Institute, Aalborg University, Aalborg, Denmark

${ }^{3}$ Neonatal Intensive Care Unit, Landspitali - The National University Hospital of Iceland, Reykjavik, Iceland

${ }^{4}$ Department of Nursing Science, University of Turku, University of Turku, Turku, Finland

\section{Correspondence}

Renée Flacking, School of Health and Welfare, Dalarna University, Högskolegatan 2, SE-791 88 Falun, Sweden.

Email: rfl@du.se

Funding information

The authors received no specific funding for this work.

\begin{abstract}
Aims and objectives: This study aimed to explore staff attitudes and experiences of parents' friends and families' social presence and involvement in neonatal intensive care units (NICUs).

Background: In NICUs, parents need emotional and practical support during infant hospitalisation. Friends and families of parents may constitute the most significant providers in this support, but few studies are available on when and how these 'important others' can be present and involved.

Design: This qualitative descriptive study was based in the philosophical tenets of naturalistic inquiry.

Methods: Seven focus groups were conducted where 67 staff from Denmark, Finland, Iceland and Sweden participated. Data were analysed using thematic analysis. The study was reported following the COREQ guidelines and checklist.

Results: The overarching theme showed that 'important others' were an unaddressed group of potential supporters in the periphery. The five identified themes described how staff recognised 'important others' as the parents' territory, but that 'important others" presence and involvement needed to be negotiated with staff. Although the staff regarded 'important others' as necessary for parents' emotional, practical and social support, they felt less obligated to support them as part of their work remit. The staff also felt that inclusion of 'important others' was an essential step forward in achieving family centred care.

Conclusions: The findings indicate that 'important others" involvement was primarily guided by proactive parents and unit care culture rather than by staff's formal written guidelines or guidance. Single-family rooms seemed to enhance the presence and involvement of 'important others'.

Relevance to clinical practice: There is a need for more staff resources to enable and support the participation of 'important others'. Parents need to be included during the
\end{abstract}


development of policies to provide their experiences. Finally, more research is needed on what parents wish from their 'important others'.

KEYWORDS

family-centred care, neonatal care, nurse-patient relationship, qualitative study, support

\section{1 | INTRODUCTION}

A key component of family centred care (FCC) in neonatal intensive care units (NICUs) is the psychosocial support offered to parents. Parents of preterm and ill infants are exposed to stress, negative feelings, anxiety, trauma and an unfamiliar environment, and for many, a prolonged parent-infant separation and an uncertain future for the infants (Flacking et al., 2012). The prevalence of posttraumatic stress disorder and depression are much higher among parents whose infants have been cared for in a NICU (Carson et al., 2015; Kim et al., 2015; Tandberg et al., 2019). Consequently, parents of preterm infants need psychosocial support and practical help during the NICU stay. Feeling well supported is crucial for the parents and for developing parent-infant relationships: well-supported parents with high self-esteem have higher levels of responsivity and attachment to their infants (Amankwaa et al., 2007).

Friends and family, hereafter referred to as 'important others', may constitute the most important emotional and practical source of support for parents. However, neonatal care research has almost exclusively focussed on the staff's support to parents and little about 'important others'. This article focusses on 'important others' from a staff perspective.

\section{2 | BACKGROUND}

Numerous studies have suggested the importance of parents' experiences of their relationship with staff. For parents of infants cared for in a NICU, the parent-staff relationship is a 'new' relationship and sensitive to intrapersonal and interpersonal factors, time, design of the NICU and the staff's mindset (Bry \& Wigert, 2019; Oude Maatman et al., 2020; Sigurdson et al., 2020). Unlike staff, family members or close friends have usually been part of the parents' life for an extended period and are persons that parents trust, share feelings with, and rely on for emotional and practical support. Thus, 'important others' constitute a valuable inspirational and practical resource during infant hospitalisation (Hagen et al., 2019). However, parents of preterm infants may experience less support than needed because family and friends do not know how to support the parents (Korja et al., 2009). One potential reason for this association is the lack of opportunities for 'important others' to be present and involved. Studies have shown that few NICUs allow 'important others' to participate in the care process without restrictions (e.g., accompanied by parents or staff agreement; Flacking et al., 2019; Greisen et al., 2009). Further, the awareness of how it is for parents

\section{What does this article contribute to the wider global clinical community?}

- 'Important others', i.e., friends and family regarded as the closest and most valuable persons, are an unaddressed group of potential supporters to those parents whose child is hospitalised.

- Single-family rooms and a supportive care culture are beneficial for facilitating 'important others" presence and involvement.

- To improve family centred care by including 'important others', staff need more resources to empower and support "important others" participation and facilitate for parents to take an active role in developing policies and guidelines to make them valid and relevant.

to become parents in a NICU may not be well understood by 'important others' and therefore emotional support is difficult to provide.

With a paradigm shift to humanise the NICU environment, parents take a more active role in care (Roue et al., 2017). Single room design provides the family with privacy and an opportunity to be with their baby in the NICU on a 24-h basis, resulting in reduced NICU-related parental stress and potentially improved neonatal morbidity and mortality (O'Callaghan et al., 2019; van Veenendaal et al., 2020). In addition, this design enable the presence and involvement of 'important others'. Because concerns have been raised that parents may become exhausted and isolated (Anderzen-Carlsson et al., 2014; Anderzen-Carlsson et al., 2014), the inclusion of 'important others' may contribute to parents' emotional health and wellbeing.

Few studies have explored the role of 'important others' in the context of neonatal care. To our knowledge, the first study about staffs', parents' and grandparents' perceptions of current visiting policies and sources of support was published in 1991 (McHaffie), which showed that professionals found grandparents to be a 'problem group' and that the existing policies for grandparents' involvement did not meet perceived needs. Most grandparents were tolerant of the restrictions limiting their access but parents were less satisfied on the grandparents behalf (McHaffie, 1991). Most studies have focussed on grandparents' experiences, where findings show that grandparents have ambivalent feelings because of having a grandchild born preterm. The grandparents feel confused while also wanting to be included and supportive (Adama et al., 2018; Brodsgaard et al., 2017; Frisman et al., 2012). By shifting the traditional care model to a FCC 
TABLE 1 Characteristics of the study participants by country

\begin{tabular}{|c|c|c|c|c|}
\hline Characteristics & Denmark & Finland & Iceland & Sweden \\
\hline \multicolumn{5}{|l|}{ Profession, $n$} \\
\hline Nurse & 13 & 21 & 9 & 8 \\
\hline Assistant nurse & & 2 & 2 & 10 \\
\hline Medical doctor & & & & 2 \\
\hline Age, years, mean (range) & $42(26-65)$ & $40(25-58)$ & $42(23-65)$ & $41(25-63)$ \\
\hline Years working in neonatal intensive care, mean (range) & $12(2-22)$ & $15(0.5-39)$ & $12(1-32)$ & $12(0.5-45)$ \\
\hline Had experience of being a parent to a baby in a NICU, $n$ & 2 & 2 & 0 & 4 \\
\hline Had been an 'important other' to a parent in a NICU, $n$ & 5 & 13 & 4 & 12 \\
\hline
\end{tabular}

approach, the family's extended network could be a major resource for parents' emotional and practical support. To our knowledge, no studies have explored the hindrances and facilitators for 'important others" presence and involvement. Moreover, research has not investigated the attitudes and experiences of NICU staff. Because staff constitute the gatekeepers of care policies, their views and perspectives are extremely important for the NICU culture. Thus, this study aimed to explore staff attitudes and their experiences of presence and involvement of 'important others' in NICUs.

\section{3 | METHODS}

\section{1 | Design and setting}

This qualitative descriptive study was based in the philosophical tenets of naturalistic inquiry (Lincoln \& Guba, 1985). Staffs were interviewed about their varied shared experiences using focus groups. Seven NICUs participated; two NICUs in Denmark, Finland and Sweden and one in Iceland. Data collection covered a 1-year period (May 2019 to May 2020). This article adheres to the COREQ checklist for reporting qualitative studies (Tong et al., 2007) (Appendix S1).

Family centered care is an established approach in the Nordic countries. Staff and parents work in partnership in infant care and parents are encouraged to stay $24 / 7$ in the units. The social security systems support a continuous stay in the units with paid parental leave for nine or more months. The Nordic NICUs have systematically been rebuilt with single-family room (SFR) architecture. The architecture of the seven participating NICUs was a combination of SFRs and open-bay rooms, where one NICU had only SFRs. All units supported the parents' presence next to the infant with reclining chairs, parental beds, or both. Six of seven NICUs had a policy concerning the visitations of 'important others'. The number of visitors at a time was limited to two in five of these policies. In three policies the restriction concerned only open bay rooms.

\section{2 | Participants}

Convenience sampling was used in the recruitment of the NICU staff. To capture variation within the phenomenon, two units were included in each country if possible. All staff members in each unit were invited to participate by e-mail or by face to face with a staff nurse. No staff member dropped out after having signed the consent form. Sixty-seven staff participated in the study: 51 (76\%) nurses, 14 (21\%) assistant nurses, and 2 (3\%) medical doctors. The nurses had a variation of qualifications where some had an undergraduate degree in nursing and some had a specialist degree in, e.g., paediatric nursing, intensive care nursing or midwifery. Assistant nurses were health professionals who provided basic care to patients and monitored their vital signs and health under the supervision of a registered nurse. The participants' age ranged from 23-65 with an average of 41 years. Most of the participants had worked for more than 10 years within neonatal care. The characteristics of the study participants are described in Table 1.

\section{3 | Research ethics}

The study protocol had favourable statements from ethical committees in Denmark (Danish Data Protection Agency, 2008-58-0028), Finland (Ethics committee at University of Turku, 35/2019), Iceland (The National Bioethics Committee, VSN-19-108) and Sweden (the Swedish Ethical Review Authority, 2019-01405). In addition, the study protocol was approved by each participating hospital according to local policy. All participants provided informed, written consent before participation and were ensured confidentiality. All data, i.e., audio files, transcripts and field notes, were stored confidentially and secure in each country in accordance with the General Data Protection Regulation.

\subsection{Data collection}

All staff members of each NICU were provided written and verbal information about the study. Those willing to participate contacted the local researcher and agreed on the time and place for the focus group interview. Interviews for two focus groups were conducted in a private room in each hospital, except in Denmark, where only one focus group was available at one of the hospitals, resulting in 13 focus groups, with 4-11 participants in each group. Only the 
TAB LE 2 Themes and subthemes to the overarching theme 'Important others'-unaddressed supporters in the periphery

\begin{tabular}{|c|c|}
\hline Themes & Subthemes \\
\hline \multirow[t]{4}{*}{ The illusive guideline of 'important others' presence } & Practice/staff-based guidelines \\
\hline & Knowledge about the existence and content of a guideline/policy \\
\hline & Gliding guideline - negotiations and flexibility dependent on reasons \\
\hline & Guidelines are there for protecting infants \\
\hline \multirow[t]{3}{*}{ Important others-parent territory by proactive negotiations } & Nurses respect parents' choice who they want to invite \\
\hline & Variations in attitudes concerning IOs presence and involvement \\
\hline & Parents' power to decide what IO can contribute with \\
\hline \multirow[t]{5}{*}{ The pros and cons of important others for parents and infants } & $\begin{array}{l}\text { IO are support for parents by being there and seeing their everyday life, and for } \\
\text { emotional and practical support }\end{array}$ \\
\hline & Showing the baby is important for becoming a parent-a sign of normality \\
\hline & IO may hurt parents' feelings and take away their attention from the baby \\
\hline & Parents needs of privacy \\
\hline & Parents wanting to protect their IOs from worry \\
\hline \multirow[t]{4}{*}{ Staff obligation to support important others } & IOs are not a top priority \\
\hline & No resources and burdensome to support IOs \\
\hline & Self-evident to support IO as a member of staff \\
\hline & Work with IOs through parents and responsibility to guide \\
\hline \multirow[t]{3}{*}{ A move forward-a shift from protection to inclusion } & Facilities for having $1 \mathrm{O}$ there much more \\
\hline & Seeing parents in a new light/context \\
\hline & Evident role in helping parents to involve IOs \\
\hline
\end{tabular}

participants and researchers were present during the focus groups. In Sweden and Denmark, the interviews were facilitated by senior researchers (RF, HHT), with extensive experience of conducting interviews. In Finland, the interviews were facilitated by a senior researcher (AA) with extensive experience of conducting interviews and a master student (SP) with some experience. In Iceland, the interviews were facilitated by two undergraduates with experience of conducting qualitative research. All interviewers were female with no acquaintances among the participants. Interviewers introduced themselves to the participants by profession and a short description of where they were working. At the beginning of the focus group interviews, NICU staff filled in a questionnaire about background information. The interviewer supported and encouraged both exploration and clarification of participants' individual and shared views and beliefs about the topic. Fieldnotes were documented during the interviews, which were audio recorded and lasted approximately 90 minutes. No member checking was conducted.

\subsection{1 | The interview guide}

The original semi-structured interview guide was developed in English by the researchers representing each country (RF, HHT, RJ, AA) and translated into the target languages (Danish, Finnish, Icelandic, Swedish). After the translation, the researchers held a cognitive debriefing to ensure that the interview questions maintained their original meaning. The semi-structured interview guide covered the following themes: (1) unit written and informal policies concerning 'important others', (2) the experiences of the staff on the roles of grandparents, relatives and friends in supporting parents and caring for the infant and (3) the responsibilities of the staff regarding 'important others' in the NICU. The first interview in each country was considered as pilot. Based on these pilot interviews, there was no need to do any changes to the interview guide, so these data were included in the analysis. Written unit policies about 'important others', if available, were collected from the participating NICUs.

\section{5 | Data analyses}

The qualitative data were analysed using thematic analysis (Braun \& Clarke, 2006). Two researchers (RF and AA) created the initial coding frame inductively in English based on the Swedish and Finnish data. HHT and RJ deductively coded Danish and Icelandic data with this initial coding frame and suggested additional inductive codes. Codes and preliminary themes were reviewed several times by the research group. Variations were noted between participants and units. Based on these discussions, the research group defined five themes and 19 subthemes and generated an initial storyline with an overarching theme (Table 2). Variations in the data were integrated into the storyline. The report was discussed several times within the research group and modified to achieve conceptual equivalence accordingly. Consensus was reached at each phase. 


\section{4 | FINDINGS}

\section{1 | 'Important others'-unaddressed supporters in the periphery}

Our findings revealed that 'important others' were an unaddressed group of potential supporters in the periphery regarding their presence and involvement. For the staff, the topic of 'important others' was new and they felt the guidelines were elusive and open to different interpretations. The staff recognised 'important others' as the parents' exclusive territory, but their presence needed to be negotiated with the staff. Although the staff regarded 'important others' as significant for parents' emotional, practical and social support, they felt less obligated to support them as part of their work remit. The staff also believed that 'important others' were helping advance FCC. The findings are presented in five themes.

\subsection{1 | The illusive guideline of 'important others' presence}

All NICUs but one had written guidelines for "important others" presence, but none about the involvement of 'important others' in the care process. Guidelines had been formulated based on the opinions of the staff and implemented without collaboration with the parents. Most staff had never participated in an in-depth discussion on guidelines and when they were asked about the guideline/policy, they became hesitant and asked each other whether such a policy existed, where to find it and what it stated: 'I know there are rules, but I don't know what they are. I would fail on it if this were a test' (ICE). Some units had transitioned during the past years in their design from open-bay rooms to SFRs and had implemented an FCC program. Guidelines for the presence of 'important others' had evolved accordingly, where most units had no restrictions in SFRs and a maximum of two visitors in open-bay rooms.

We wrote a new policy 5-6 years ago when we moved to this new unit [single-family rooms and a few open-bay rooms for intensive care]. We aimed to have the parents, siblings and the whole family in the single units. However, we soon realised that it could get crowded in intensive care rooms if there were 3-4 infants in incubators with many visitors, so that's why we have a rule of two visitors per family in those rooms. (SWE)

In SFRs parents had more power to decide how many visitors and when, they could visit, although these decisions had to be negotiated with the staff. One nurse described an overarching principle: 'Own room - own decisions' (SWE). In most units the maximum number of visitors in open-bay areas was restricted to two persons per family for each visit. However, that restriction could be negotiated depending on circumstances, such as other families' presence, the infant's medical status and the anticipated length of hospital stay.

Well, it depends on the season, too. If we have a full unit and two children in the room or if the children are in the open bay, we have to think about the size of the 'visitor crowd'. It is on a case-by-case basis. Younger couples wish for all their friends to visit. That is something that we need to discuss. (FIN)

Also, the flexibility was described as a personal choice in that the staff could choose not to follow the policy if they believed it to be too restrictive or not conducive to the parents' wellbeing: 'I am awful in breaking the rules. I just... I just do what I feel is right. I have a real problem rejecting parents' request to bring someone to the unit' (ICE).

In all NICUs, the staff stated that the primary purpose for having guidelines was to 'protect' the infant in the NICU, especially infants in open-bay rooms. By protection, the staff meant reducing the risk for confidential breach of trust, infections and excessive noise. Moreover, by having many visitors, the staff experienced that they sometimes lost focus on the infants or that the work situation became more challenging because of cramped space, which reduced the staff's ability to quickly interact if needed. One nurse said, 'I was going to change fluids and I just couldn't access the incubator because there were way too many visitors and dividers' (ICE). Hence, in some units the staff wanted more transparent rules and limitations.

Many visitors at the same time create chaos. It also destroys our relationship with the families because we cannot get the parents' attention as they are so occupied with visitors. Furthermore, there might be more private things we do not want to address in front of others. In reality, it might be better without visits at all in the open bay rooms. (DEN)

\subsection{2 | 'Important others'-parent territory by proactive negotiations}

Across NICUs, the parents could invite who they wanted to the NICU. For all NICUs, the staff always showed respect for the parents' preferences.

We don't care whether it's relatives, friends or neighbours. It's the parents who decide on whom they want to come and see as 'important others'. We tell them that early on, and then they will just let us know beforehand so that it's feasible. (SWE)

Although the staff in some units described how they encouraged parents to invite their 'important others', most parents had to be proactive in their actions in asking staff: 'Mostly the parents ask if it 
is okay for the grandparents to come and then we say, 'yeah, okay'. (FIN).

There were large variations in attitudes among the NICUs concerning 'important others" involvement in care. These differences seemed to be associated with the unit's design, the parents, and the care culture. In one NICU parents were told that they could be in the NICU 24/7 and preferably with one of the parents always remaining with the infant. Hence, the staff were positive towards the involvement of 'important others', as parents sometimes needed support or for someone to temporarily take over the infant's care. In another NICU parents could only visit and 'important others' were only allowed to hold the baby as the staff wanted the infants to bond with the parents. Regardless of the setting, it was always the parent's choice, within the stated limitations, on what role 'important others' should play. There were no restrictions in most NICUs on the involvement of 'important others' (e.g. have skin-to-skin contact, tube or bottle feed, change diapers) if the parents agreed.

\subsubsection{The pros and cons of 'important others' for parents and infants}

The staff experienced that friends and family supported parents by coming to the NICU and seeing the parents' everyday life, which would bring them close to each other and enable 'important others' to understand the parents' situation better.

It's like full-term sick infants [infants with, e.g., congenital heart defects and diaphragm hernias] need so much. I have often seen grandmas and grandpas step up so well and it's so beautiful and precious to see. (ICE)

Some staff emphasised the importance of enabling the normal process of becoming a parent, which meant that parents could show their infant and talk about the infant with their 'important others'. The staff pointed out that the mobile phone facilitated this process.

Mobile phones are their [parents] link to the outside world. When grandma doesn't have the possibility or can't afford to travel, parents can contact their friends. They can see each other and talk [on a social media platform], and you can see that it makes them happy. They have shown their baby and the environment. (SWE)

Staff also described the importance of practical support, such as baby-sitting older siblings or cooking for the parents, which allowed the parents the possibility to remain in the NICU.

I don't think it necessarily supports parents' needs [in the NICU], but [it does] very much supports the family. Suppose they, for instance, have other children at home. And that's where I see that the support from the network is vital. (DEN)

The staff described that "important others" presence enabled the normalisation of the situation in that parents had someone they could be with (e.g., talking, going shopping or visiting a café). "Important others" presence and involvement also permitted others to bond with the infant during the NICU, facilitating receiving help with the infant after discharge.

The staff also noted some negative aspects of 'important others" presence and involvement; they, as staff, wanted to protect the parents. Some staff members explained that they had experienced that an important other had hurt the parents' feelings, causing the parents psychological stress and symptoms of exhaustion. For all NICUs, staff experienced that some 'important others' had 'taken over', hindering the parents from performing routine parent tasks. Other staff members remarked that the mere presence of 'important others', especially in the first days or weeks, was a disturbance.

We encourage parents that don't want to have visitors in the beginning because it's not good when so many stand near the mother when she's trying to breastfeed. It's not good when many people come [to visit], as it is disturbing. It is much easier for parents if there are no visits. (DEN)

Some staff highlighted that the presence of 'important others' might reduce parents' attention to their infant, which the staff identified as a potentially harmful practice.

Across all NICUs, staff were unsure about how to protect parents from what they believed to be harmful or exhausting comments and actions by 'important others'. One nurse described a situation where she had entered a SFR where friends had joined the parents. The room was noisy, and the infant seemed to be unwell. The male friend was helping the mother with breastfeeding.

I did not know what to do. And I said, 'Okay, you have been here for a couple of hours now. Visitors are allowed, but [they're] mainly the parent's grandparents and siblings. Friends can have a quick visit'. They understood and left shortly after. And the mom changed [her behaviour] and started to focus on the baby. (FIN)

Some staff noted that it was much easier to react appropriately if they had a trustful relationship with the parents and talked about their 'important others'.

It takes some work to make the parents feel at ease. We also have a sensitive ear and listen to how parents think so that their 'important others' don't take over. Thus, we support parents in the best way. You need 
time for that [to develop] and the ability to be there.

(SWE)

The staff reported that the parents' need for privacy was the leading reason they did not invite 'important others' to the NICU. Some staff recounted that parents wanted to protect their 'important others' from worries. In those cases, the staff saw themselves as gatekeepers and acted on behalf of the parents: 'Some parents struggle to say 'no,' and then I tell the parents to say to us so that we can be the bad guys, by blaming it on a temporary visiting ban or something else'. (SWE).

\subsection{4 | Staff obligation to support 'important others'}

In all NICUs, the staff outlined that the presence and involvement of 'important others' were not top priorities. However, there were some differences between staffs in different NICUs in their beliefs and attitudes towards helping 'important others'. Some experienced that they had no resources to oversee 'important others' and felt that parents' presence and involvement was sufficient. The staff thought that it was burdensome if there were too many grown-ups visiting or that 'important others' were an obstacle or hindrance to performing their duties. On the other hand, some NICU staff, especially those with a longer history of FCC, found it easier to include and support 'important others'. The staff in those units expressed it as self-evident to support 'important others' and did not experience 'important others" presence and involvement as troublesome. However, different staff teams were not always in agreement. For instance, one nurse stated, 'If we are supposed to teach many people on how to take care of the infant, it's too much work for us and things will not be done as they should be'. (ICE) Another staff member felt a responsibility to support the presence of 'important others'.

For most parents, it is priceless to get support from family or friends. It's also important for us nurses, as I see it, to involve 'important others'. I mean to get the parents to involve them ['important others']. (ICE)

Regardless of NICU culture, the staffs agreed that information to 'important others' and the support to them should only be given according to the parents' preferences. Staffs described that they wanted validation from parents on what to say concerning the infant but that they provided information more freely on general care and equipment.

I give the parents space to say the things that they want about the baby and their situation. I withdraw in a way; I'm not there talking like I would to the parents about the baby's current condition. It's the parents that tell us what they want. And if I'm in the room and they ask something, then of course I answer. (FIN)
If parents wanted their 'important others' to be involved, staff supported the 'important others' just as they would support the parents, i.e., showing and guiding them through the task.

During the first visits by grandparents, you feel that they don't know prematurity, tube feeding, etc. But when they've been here a few times and we informed them, things go much better. (DEN)

\subsection{5 | A move forward-a shift from protection to inclusion}

For all participating NICUs, staff reported a shift to a more family centred approach. In NICUs with limited space, the main concern was to facilitate more presence for the parents. Staff in NICUs with SFRs experienced that there were additional improvements to create a more family centred setting.

We are now in the phase where we reunite the baby with the family but create a separation between parents and their normal network. And that's a problem. There is another side to every coin and with singlefamily rooms, we risk isolating the parents from their network given that they don't leave the unit. Still, I experience parents less isolated in the single-family rooms because they receive visits. It's not unusual that both parents are in bed having skin-to-skin with their baby and with grandma in the bed too. (SWE)

Many staff members mentioned a crash of norms that became particularly evident when 'important others' were present or involved in the care. By having 'important others' present, they saw more of the parents' uniqueness and cultural beliefs and practices than before. When parents were either visited by 'important others' or involved in the care process, the staff sometimes saw that the parents behaved differently than what they had done when only in the staff's presence. The staff saw the parents in a new light, which baffled or confronted them with the differences in norms on how to parent, communicate and behave. For some staff, this caused stress or feelings of exclusion. Others emphasised that they wanted to learn more.

One commonly mentioned strategy to include 'important others' was encouraging parents to invite their 'important others' to the NICU. One NICU had written information to 'important others' and that one NICU also had a 'grandparent café', where all 'important others' could join. Suggestions were also made on developing a plan together with parents on how to involve their 'important others'. The nurse had an essential role in helping parents involving their 'important others' during the hospital stay or preparing for discharge.

If we consider the family's network's involvement as a quality in our work, there has to be a policy 
supporting that position, and we should document our work. It is not enough that each nurse determines whether she thinks this is important. We must make it an integrated part of the systematic work with the families, which is always integrated as a quality of our neonatal care. (DEN)

\section{5 | DISCUSSION}

In this qualitative study, staff were interviewed about their shared experiences of social presence and involvement by 'important others' in NICUs, using focus groups in four countries. Our findings show that staff attitudes and experiences of "important others" presence and involvement varied within and between the countries. 'Important others' involvement was primarily guided by proactive parents and a unit care culture, not supported by the staffs' formal written guidelines and guidance. The discussion focuses on identifying facilitating factors for involvement of 'important others', the benefits of involvement perceived by staff and the staff's potential needs for resources and education.

Many NICUs pose restrictions on the presence of 'important others'. Previous studies have shown that most units allow grandparents and friends' visits under conditions, such as parents accompanying grandparents (Greisen et al., 2009), a maximum of two visitors per day or visits limited to $2 \mathrm{~h}$ (Flacking et al., 2019). Our findings suggest that the most direct influential factor for 'important others" presence and involvement is the unit architecture. Single-room design allows unrestricted access to the unit, privacy and autonomy for the family (Lester et al., 2014; Makela et al., 2018; Toivonen et al., 2017). In such an environment 'important others' do not disturb or disrupt other families in the unit and provide much-needed social support for those who might otherwise feel isolated and lonely in a hospital setting (Anderzen-Carlsson, Lamy, \& Eriksson, 2014; Anderzen-Carlsson, Lamy, Tingvall, et al., 2014).

Although a unit's design and architecture are important in the care sector, it constitutes only a 'space and place' to provide care. NICUs' care culture might be covert and difficult to determine which elements comprise the care culture. FCC in NICUs is a developing concept that has gradually changed over time. Today, FCC principles are suggested to include 24-h parental access to infants, psychological support, pain management, supportive environment, postural support, skin-to-skin contact, breastfeeding and lactation support, and sleep protection (Roue et al., 2017). Our findings suggested that staff had ambivalent feelings about the involvement of 'important others'. The ambiguous and somewhat undefined nature of this involvement was expressed when staff described 'important others' as the parents' territory, and yet their presence needed to be negotiated with staff. FCC's definition must evolve towards a more holistic and realistic view on families in a relational context of 'important others' to enable them to have a more active role in supporting parents and providing care for the infant in the NICU. FCC in the NICU has traditionally focussed on the two-generation family structure, i.e., infant and parents (Mikkelsen \& Frederiksen, 2011). Thus, minimal attention has been given to the involvement of grandparents, friends and siblings. The definition of FCC in a paediatric context can help broaden our perspective and explore the possibilities of care provided more closely in the context of families' social networks (Kuo et al., 2012; Shields et al., 2006, 2007).

Involving 'important others' in the infant's care should be a marker of quality in neonatal care. The positive benefits of parents receiving support have been illuminated by Hagen et al. (Hagen et al., 2019), who showed that help from family and friends is a crucial sociodemographic attribute associated with parental satisfaction. Studies have also shown that being unable to share their baby with other family members is a primary source of stress for parents of NICU infants (Shaw et al., 2006). Studies have further shown that supportive networks reduce maternal depression and enhance parenting competence (Davis et al., 2003). These findings indicate that parents benefit psychologically from a caring and dynamic network during the infant's hospitalisation. Therefore, the NICU staff must be willing to alter their care practices to provide autonomy for parents to include and involve 'important others' in the infant's care.

A move towards a more inclusive FCC requires a shift in mindset and care culture. Nurses serve as vital stakeholders in facilitating 'important others" involvement in daily practise in the NICU. To achieve this goal, nurses require resources, support, education, and continual feedback. Our findings show that the NICUs did not have guidelines and protocols to guide the involvement of 'important others'. A starting point would be to initiate discussions, educate staff on supporting 'important others' and formulate policies. By including parents in these activities, contextual facilitating and hindering factors can be identified, enhancing the validity and relevance of the guidelines. It is also crucial that all staff are accessible (nurses, doctors, therapists and other health care workers) when incorporating 'important others' into the care process (Axelin et al., 2014).

Little knowledge is available on how parents prefer to involve 'important others' in their infant's hospital care. We have identified only one published study showing that parents' wanted emotional support from grandparents and being engaged in the care process (McHaffie, 1991). There are few studies on the role of 'important others' in the NICU. The few existing studies have mainly focussed on the grandmothers' desire to be supportive, engaged and involved in the care of families but also concerned about disturbing or being intrusive (Frisman et al., 2012; Hall, 2004; Lindberg \& Ohrling, 2008; Ravindran \& Rempel, 2011). The knowledge gap in improving care and parents' situation in relation to 'important others' needs to be addressed in future studies.

\section{1 | Strengths and limitations}

From the beginning of the study, the research group engaged in a reflexive research process, i.e., a continuous examination of the data 
collection, data, researchers/participants and the research context. During the first meetings, a pre-understanding of the phenomenon under investigation was created and noted. The researchers tended to favour parent perspectives, and at times, it was challenging to be open to the nurses' attitudes. This understanding was revisited and discussed during the data collection, analysis and writing process. Awareness about the pre-understanding suggests that the findings are authentic in describing the participants' feelings and wishes. Credibility of the findings was supported by data saturation, which emerged in the last focus group in each country where no new relevant data appeared. However, practices related to 'important others' and FCC implementation varied between and within the Nordic countries in terms of, for example, ratio of SFRs and presence of parents. Due to convenience sampling, it could be expected that those participating were more favourable towards 'important others'. However, the variations in data with positive and negative statements towards 'important others' indicate that those participating were likely to represent broad variations of perceptions. Thus, the broader applicability and transferability of the findings to other settings should be evaluated in the light of this variation. A rigorously created and translated semi-structured interview guide and coding frame (audit trail) supported the dependability of the results.

\section{6 | CONCLUSIONS}

Our findings show that 'important others' are an unaddressed group of potential supporters that currently lack presence and involvement in the care process. 'Important others" involvement is guided by proactive parents and a unit care culture rather than by the staff's formal written guidelines and guidance. We observed that single-family rooms and a supportive care culture are beneficial for facilitating 'important others" presence and involvement.

\section{7 | RELEVANCE TO CLINICAL PRACTICE}

Involving 'important others' in the infant's care should be a marker of quality in neonatal care. To improve FCC by including 'important others', staff need more resources to empower and support 'important others' participation. The initiation of discussions on how staff can support 'important others' and formulate policies for the NICU is a starting point. By including parents in these activities, contextual facilitating and hindering factors can be identified, enhancing the validity and relevance of the guidelines. Finally, more research is needed on what parents require from their 'important others' and how they can support parents and infants.

\section{ACKNOWLEDGEMENTS}

We want to acknowledge Jóhanna Ósk Tryggvadóttir, Birna Rut Aðalsteinsdóttir and Christine Breili, for their support with the data collection process. We also want to acknowledge the SCENE Network for the early contributions in designing the study.

\section{CONFLICT OF INTEREST}

The authors have no conflict of interest to declare.

\section{AUTHOR CONTRIBUTIONS}

The study was designed by RF, HHT, RJ and AA. All authors participated in the data collection and analyses, RF wrote the original draft, all authors commented on the manuscript and all authors read and approved the final manuscript.

\section{ORCID}

Renée Flacking (D) https://orcid.org/0000-0002-4013-1553

\section{REFERENCES}

Adama, E. A., Bayes, S., \& Sundin, D. (2018). Parents' experiences of caring for preterm infants after discharge with grandmothers as their main support. Journal of Clinical Nursing, 27(17-18), 3377-3386. https://doi.org/10.1111/jocn.13868

Amankwaa, L. C., Picklet, R. H., \& Boonmeet, J. (2007). Maternal responsiveness in mothers of premature infants. Newborn and Infant Nursing Reviews, 7(1), 25-30.

Anderzen-Carlsson, A., Lamy, Z. C., \& Eriksson, M. (2014). Parental experiences of providing skin-to-skin care to their newborn infantpart 1: A qualitative systematic review. International Journal of Qualitative Studies on Health and Well-being, 9, 24906. https://doi. org/10.3402/qhw.v9.24906

Anderzen-Carlsson, A., Lamy, Z. C., Tingvall, M., \& Eriksson, M. (2014). Parental experiences of providing skin-to-skin care to their newborn infant-part 2: A qualitative meta-synthesis. International Journal of Qualitative Studies on Health and Well-being, 9, 24907. https://doi.org/10.3402/qhw.v9.24907

Axelin, A., Ahlqvist-Bjorkroth, S., Kauppila, W., Boukydis, Z., \& Lehtonen, L. (2014). Nurses' perspectives on the close collaboration with parents training program in the NICU. MCN, American Journal of Maternal Child Nursing, 39(4), 260-268. https://doi.org/10.1097/ NMC.0000000000000061

Braun, V., \& Clarke, V. (2006). Using thematic analysis in psychology. Qualitative Research in Psychology, 3, 77-101. https://doi. org/10.1191/1478088706qp063oa

Brodsgaard, A., Helth, T., Andersen, B. L., \& Petersen, M. (2017). Rallying the troops: How sharing knowledge with grandparents supports the family of the preterm infant in neonatal intensive care unit. Advances in Neonatal Care, 17(3), E1-E10. https://doi.org/10.1097/ ANC. 0000000000000360

Bry, A., \& Wigert, H. (2019). Psychosocial support for parents of extremely preterm infants in neonatal intensive care: A qualitative interview study. BMC Psychology, 7(1), 76. https://doi.org/10.1186/ s40359-019-0354-4

Carson, C., Redshaw, M., Gray, R., \& Quigley, M. A. (2015). Risk of psychological distress in parents of preterm children in the first year: evidence from the UK millennium cohort study. British Medical Journal Open, 5(12), e007942. https://doi.org/10.1136/bmjop en-2015-007942

Davis, L., Edwards, H., Mohay, H., \& Wollin, J. (2003). The impact of very premature birth on the psychological health of mothers. Early Human Development, 73(1-2), 61-70. https://doi.org/10.1016/ S0378-3782(03)00073-2

Flacking, R., Breili, C., \& Eriksson, M. (2019). Facilities for presence and provision of support to parents and significant others in neonatal units. Acta Paediatrica, 108(12), 2186-2191. https://doi. org/10.1111/apa.14948

Flacking, R., Lehtonen, L., Thomson, G., Axelin, A., Ahlqvist, S., Moran, V. H., Ewald, U., \& Dykes, F. (2012). Closeness and separation in 
neonatal intensive care. Acta Paediatrica, 101(10), 1032-1037. https://doi.org/10.1111/j.1651-2227.2012.02787.x

Frisman, G. H., Eriksson, C., Pernehed, S., \& Morelius, E. (2012). The experience of becoming a grandmother to a premature infant - a balancing act, influenced by ambivalent feelings. Journal of Clinical Nursing, 21(21-22), 3297-3305. https://doi. org/10.1111/j.1365-2702.2012.04204.x

Greisen, G., Mirante, N., Haumont, D., Pierrat, V., Pallás-Alonso, C., Warren, I., \& Cuttini, M. (2009). Parents, siblings and grandparents in the Neonatal Intensive Care Unit. A survey of policies in eight European countries. Acta Paediatrica, 98(11), 1744-1750.

Hagen, I. H., Iversen, V. C., Nesset, E., Orner, R., \& Svindseth, M. F. (2019). Parental satisfaction with neonatal intensive care units: a quantitative cross-sectional study. BMC Health Services Research, 19(1), 37. https://doi.org/10.1186/s12913-018-3854-7

Hall, E. O. (2004). A double concern: Grandmothers' experiences when a small grandchild is critically ill. Journal of Pediatric Nursing, 19(1), 61-69. https://doi.org/10.1016/s0882-5963(03)00143-x

Kim, W. J., Lee, E., Kim, K. R., Namkoong, K., Park, E. S., \& Rha, D. W. (2015). Progress of PTSD symptoms following birth: A prospective study in mothers of high-risk infants. Journal of Perinatology, 35(8), 575-579. https://doi.org/10.1038/jp.2015.9

Korja, R., Savonlahti, E., Haataja, L., Lapinleimu, H., Manninen, H., Piha, J., \& Lehtonen, L. (2009). Attachment representations in mothers of preterm infants. Infant Behavior and Development, 32(3), 305-311. https://doi.org/10.1016/j.infbeh.2009.04.003

Kuo, D. Z., Houtrow, A. J., Arango, P., Kuhlthau, K. A., Simmons, J. M., $\&$ Neff, J. M. (2012). Family-centered care: Current applications and future directions in pediatric health care. Maternal and Child Health Journal, 16(2), 297-305. https://doi.org/10.1007/s1099 5-011-0751-7

Lester, B. M., Hawes, K., Abar, B., Sullivan, M., Miller, R., Bigsby, R., Laptook, A., Salisbury, A., Taub, M., Lagasse, L. L., \& Padbury, J. F. (2014). Single-family room care and neurobehavioral and medical outcomes in preterm infants. Pediatrics, 134(4), 754-760. https:// doi.org/10.1542/peds.2013-4252

Lincoln, Y., \& Guba, E. (1985). Naturalistic inquiry. Sage Publications.

Lindberg, B., \& Ohrling, K. (2008). Experiences of having a prematurely born infant from the perspective of mothers in northern Sweden. International Journal of Circumpolar Health, 67(5), 461-471. https:// doi.org/10.3402/ijch.v67i5.18353

Makela, H., Axelin, A., Feeley, N., \& Niela-Vilen, H. (2018). Clinging to closeness: The parental view on developing a close bond with their infants in a NICU. Midwifery, 62, 183-188. https://doi. org/10.1016/j.midw.2018.04.003

McHaffie, H. E. (1991). Neonatal intensive care units: visiting policies for grandparents. Midwifery, 7(4), 193-203. https://doi.org/10.1016/ S0266-6138(05)80198-6

Mikkelsen, G., \& Frederiksen, K. (2011). Family-centred care of children in hospital - a concept analysis. Journal of Advanced Nursing, 67(5), 1152-1162. https://doi.org/10.1111/j.1365-2648.2010.05574.x

O'Callaghan, N., Dee, A., \& Philip, R. K. (2019). Evidence-based design for neonatal units: A systematic review. Maternal Health, Neonatology and Perinatology, 5, 6. https://doi.org/10.1186/s40748-019-0101-0

Oude Maatman, S. M., Bohlin, K., Lillieskold, S., Garberg, H. T., UitewaalPoslawky, I., Kars, M. C., \& van den Hoogen, A. (2020). Factors influencing implementation of family-centered care in a neonatal intensive care unit. Frontiers in Pediatrics, 8, 222. https://doi. org/10.3389/fped.2020.00222
Ravindran, V. P., \& Rempel, G. R. (2011). Grandparents and siblings of children with congenital heart disease. Journal of Advanced Nursing, 67(1), 169-175. https://doi.org/10.1111/j.1365-2648.2010.05482.x

Roue, J. M., Kuhn, P., Lopez Maestro, M., Maastrup, R. A., Mitanchez, D., Westrup, B., \& Sizun, J. (2017). Eight principles for patient-centred and family-centred care for newborns in the neonatal intensive care unit. Archives of Disease in Childhood Fetal and Neonatal Edition, 102(4), F364-F368. https://doi.org/10.1136/archdischild-2016-312180

Shaw, R. J., Deblois, T., Ikuta, L., Ginzburg, K., Fleisher, B., \& Koopman, C. (2006). Acute stress disorder among parents of infants in the neonatal intensive care nursery. Psychosomatics, 47(3), 206-212. https://doi.org/10.1176/appi.psy.47.3.206

Shields, L., Pratt, J., Davis, L. M., \& Hunter, J. (2007). Family-centred care for children in hospital. Cochrane Database of Systematic Reviews, 24(1), CD004811. https://doi.org/10.1002/14651858.CD004811.pub2

Shields, L., Pratt, J., \& Hunter, J. (2006). Family centred care: A review of qualitative studies. Journal of Clinical Nursing, 15(10), 1317-1323. https://doi.org/10.1111/j.1365-2702.2006.01433.x

Sigurdson, K., Profit, J., Dhurjati, R., Morton, C., Scala, M., Vernon, L., Randolph, A., Phan, J. T., \& Franck, L. S. (2020). Former NICU families describe gaps in family-centered care. Qualitative Health Research, 30(12), 1861-1875. https://doi.org/10.1177/10497 32320932897

Tandberg, B. S., Flacking, R., Markestad, T., Grundt, H., \& Moen, A. (2019). Parent psychological wellbeing in a single-family room versus an open bay neonatal intensive care unit. PLoS One, 14(11), e0224488. https://doi.org/10.1371/journal.pone.0224488

Toivonen, M., Lehtonen, L., Loyttyniemi, E., \& Axelin, A. (2017). Effects of single-family rooms on nurse-parent and nurse-infant interaction in neonatal intensive care unit. Early Human Development, 106107, 59-62. https://doi.org/10.1016/j.earlhumdev.2017.01.012

Tong, A., Sainsbury, P., \& Craig, J. (2007). Consolidated criteria for reporting qualitative research (COREQ): a 32-item checklist for interviews and focus groups. International Journal for Quality in Health Care, 19(6), 349-357. https://doi.org/10.1093/intqhc/mzm042

van Veenendaal, N. R., van Kempen, A. A. M. W., Franck, L. S., O'Brien, K., Limpens, J., van der Lee, J. H., van Goudoever, J. B., \& van der Schoor, S. R. D. (2020). Hospitalising preterm infants in single family rooms versus open bay units: A systematic review and meta-analysis of impact on parents. EClinicalMedicine, 23, 100388, https://doi.org/10.1016/j.eclinm.2020.100388

\section{SUPPORTING INFORMATION}

Additional supporting information may be found in the online version of the article at the publisher's website.

How to cite this article: Flacking, R., Haslund-Thomsen, H., Jónsdóttir, R., Poropudas, S., \& Axelin, A. (2021). Parents' friends and families in neonatal intensive care units: $A$ cross-national qualitative study on staff perceptions and experiences. Journal of Clinical Nursing, 00, 1-10. https://doi. org/10.1111/jocn.16139 